\title{
Identification algorithm of switched systems based on generalized auxiliary model
}

\author{
WANG Hongwei ${ }^{1,2, *}$ and XIA Hao ${ }^{1}$ \\ 1. School of Control Science and Control Engineering, Dalian University of Technology, Dalian 116024, China; \\ 2. School of Electrical Engineering, Xinjiang University, Urumqi 830036, China
}

\begin{abstract}
For switched linear system with colored measurement noises, the identification difficulties of this system are that there exist unknown switching information, unknown middle variables and noise terms in the information vector. For the mentioned issues, the fuzzy clustering and the multi-innovation recursive identification algorithm are used to deal with these problems. Firstly, the mode detection is transformed into the detection of membership degree values confirmed by the fuzzy clustering method, and the problem of mode detection is solved by judgment and decision of the fuzzy membership values. Moreover, the multi-innovation recursive identification algorithm based on the generalized auxiliary model is proposed to estimate the parameters of the switched linear system with colored noises. Finally, the effectiveness of the proposed method is verified by the results of the simulation example.
\end{abstract}

Keywords: switched linear system, fuzzy clustering, parameter estimation, multi-innovation.

DOI: $10.21629 / J S E E .2019 .06 .16$

\section{Introduction}

A hybrid system refers to the same system that simultaneously includes continuous-time dynamic subsystems and discrete-time dynamic subsystems. The switched system is an important class of hybrid systems. There are a lot of switched systems in many areas, such as industrial production systems [1], traffic control [2], and computer integrated manufacturing systems [3]. Since the switched system was put forward, it has aroused the interest of many scholars in the control field.

The switched linear system is usually composed of multiple subsystems, switched modes and switched rules. Therefore, the input/output data pairs collected from the system no longer come from a single dynamic system; instead, the sampled data sequences are constructed from the

\footnotetext{
Manuscript received January 04, 2018.

*Corresponding author.

This work was supported by the National Natural Science Foundation of China (61863034).
}

mixed outputs of the different subsystems. For the identification of switched linear system with unknown switching modes, it is necessary to confirm which operation mode to be activated by using the current sampled data points. This process is usually referred to mode detection (MD). Then, after the MD has been confirmed, the parameters of each subsystem are estimated and this process is usually referred to parameter identification (PI). Therefore, for the switched systems, there are identification difficulties as: i) how to ensure the correctness of MD which can directly affect the estimation performance of the identification method; ii) how to design a robust identification algorithm.

Aiming at the identification issue of switched linear systems (SLS), some researchers have begun to study the related work. In general, MD is a key issue which is related with the identification performance. The early work is mostly concerned with MD under the off-line way, including clustering approach [4], bounded-error based approach [5], algebraic approach [6,7], mixed integer programming approach [8], and Bayesian approach [9,10]. In [11], the subspace method was proposed to deal with the identification of SLS under the off-line way. In [11], the switching modes were assumed to be known and the various subsystems were combined to be estimated by the subspace technique. In [12], the subspace projection based on orthogonal decomposition was adopted to judge which mode is switched. A novel clustering technique was presented to solve MD by calculating the errors between the samples and the output of each mode in [13]. For the online identification of SLS, some researchers have given attention to this problem and have demonstrated their results. In [14], a recursive learning method was employed to identify the unknown parameters of the switched auto-regressive models with eXogenous input (ARX model), and the exponential convergence was proved by the theorem method. Recently, the online identification problem was studied by 
using an online MD method and a recursive identification algorithm in [15]. In [16], convex optimization and orthogonal projection algorithms were employed to study identification problems of SLS. In [17], the adaptive estimation method was proposed to improve the identification performance by adjusting the covariance matrix derived from the input information vector. In [18], there was an innovation process which is white if and only if experimental data was generated by the auto-regressive moving average model (ARMA). Based on this observation, a procedure is developed to identify the switching time.

The motivation of our paper is to deal with MD and PI of switched linear systems with colored measurement noises. The innovative contribution points in this paper are embodied as follows:

(i) The on-line fuzzy clustering method is proposed to deal with the problem of MD. The fuzzy sets can be automatically generated by fuzzy clustering. The problem of MD is solved by judgment and decision of fuzzy membership values.

(ii) According to the multi-innovation modeling principle, a multi-innovation identification algorithm based on the generalized auxiliary model is employed to estimate the unknown parameters of switched systems. Compared with the single innovation algorithm, the proposed method can remarkably improve the identification performance.

Our paper is organized as follows. Section 2 formulates the identification problems of the switched linear system with colored noises. Section 3 indicates the MD method. This section mainly introduces the online fuzzy clustering method and its application in MD. Section 4 presents a multi-innovation recursive identification algorithm to identify the parameters of SLS. Section 5 presents the simulation example to demonstrate the effectiveness. Finally, Section 6 offers some concluding remarks.

\section{Problem formulation}

In this paper, we consider the following switched discretetime system:

$$
y(t)=\frac{B^{\delta_{t}}(z)}{A^{\delta_{t}}(z)} u(t)+w(t)
$$

where $u(t)$ is the input signal, $y(t)$ is the output signal, $A^{i}(z)$ and $B^{i}(z)$ are the polynomials of known orders $\left(n_{a}^{i}, n_{b}^{i}\right), \delta_{t}$ is a piecewise switching time function satisfying $\delta_{t} \in i=\{1,2, \ldots, c\}$ in which $c$ is the total number of switching modes, $w(t)$ is a colored noise signal, and is expressed as follows:

$$
w(t)=D(z) v(t)
$$

where $D(z)$ is the polynomial of known orders $n_{d}$, and $v(t)$ is a white noise signal with zero mean and variance $\sigma_{v}$.

In the above equations, $A^{i}(z), B^{i}(z)$ and $D(z)$ are written as

$$
\begin{gathered}
A^{i}(z)=1+a_{1}^{i} z^{-1}+\cdots+a_{n_{a}^{i}}^{i} z^{-n_{a}^{i}} \\
B^{i}(z)=b_{1}^{i} z^{-1}+b_{2}^{i} z^{-2}+\cdots+b_{n_{b}^{i}}^{i} z^{-n_{b}^{i}} \\
D(z)=1+d_{1} z^{-1}+d_{2} z^{-2}+\cdots+d_{n_{d}} z^{-n_{d}}
\end{gathered}
$$

where $i=1,2, \ldots, c, z^{-1}$ is the unit backward shift operator, i.e., $z^{-1} y(t)=y(t-1)$.

Combining (1) and (2) can give the following model:

$$
y(t)=\frac{B^{i}(z)}{A^{i}(z)} u(t)+D v(t), \quad i=1,2, \ldots, c .
$$

Define the middle variable,

$$
x(t)=\frac{B^{i}(z)}{A^{i}(z)} u(t), \quad i=1,2, \ldots, c .
$$

According to (4), (3) is also written as

$$
y(t)=x(t)+D v(t) .
$$

Define the parameter vectors and the input vectors of the $i$ th subsystem, respectively,

$$
\begin{gathered}
\boldsymbol{\theta}_{i}:=\left[\begin{array}{c}
\boldsymbol{\theta}_{i s} \\
\boldsymbol{\theta}_{n}
\end{array}\right] \in \mathbf{R}^{n_{a}^{i}+n_{b}^{i}+n_{d}} \\
\boldsymbol{\varphi}^{i}(t):=\left[\begin{array}{c}
\boldsymbol{\varphi}_{s}^{i}(t) \\
\boldsymbol{\varphi}_{n}(t)
\end{array}\right] \in \mathbf{R}^{n_{a}^{i}+n_{b}^{i}+n_{d}}
\end{gathered}
$$

where $i=1,2, \ldots, c$,

$$
\begin{gathered}
\boldsymbol{\theta}_{i s}:=\left[a_{1}^{i}, a_{2}^{i}, \ldots, a_{n_{a}^{i}}^{i}, b_{1}^{i}, b_{2}^{i}, \ldots, b_{n_{b}^{i}}^{i}\right]^{\mathrm{T}} \in \mathbf{R}^{n_{i}} \\
\boldsymbol{\theta}_{n}:=\left[d_{1}, d_{2}, \ldots, d_{n_{d}}\right]^{\mathrm{T}} \in \mathbf{R}^{n_{d}} \\
\boldsymbol{\varphi}_{s}^{i}(t):=\left[-x(t-1),-x(t-2), \ldots,-x\left(t-n_{a}^{i}\right),\right. \\
\left.u(t-1),-u(t-2), \ldots,-u\left(t-n_{b}^{i}\right)\right]^{\mathrm{T}} \in \mathbf{R}^{n_{i}} \\
\boldsymbol{\varphi}_{n}(t):=\left[v(t-1), v(t-2), \ldots, v\left(t-n_{d}\right)\right]^{\mathrm{T}} \in \mathbf{R}^{n_{d}} .
\end{gathered}
$$

Using (4) and (5), they can be arranged as

$$
\begin{gathered}
x(t)=\left(\boldsymbol{\varphi}_{s}^{i}(t)\right)^{\mathrm{T}} \boldsymbol{\theta}_{i s} \\
y(t)=\left(\boldsymbol{\varphi}^{i}(t)\right)^{\mathrm{T}} \boldsymbol{\theta}_{i}+v(t) .
\end{gathered}
$$

In [19], we proposed the label functions to simplify the compution load. In this paper, we still use the label functions to improve the identification performance. The dynamic diagram of the label functions are shown in Fig. 1. 


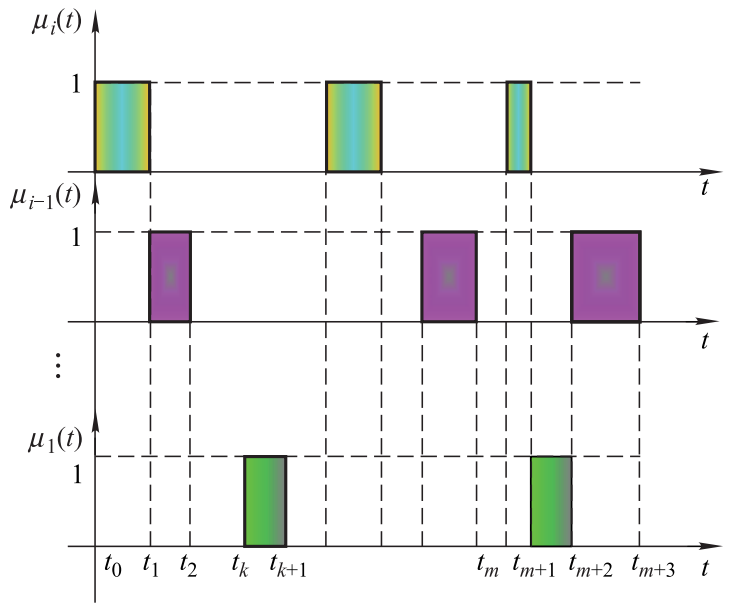

Fig. 1 Dynamic diagram of the label functions

Using the label functions in [19], (6) and (7) can be rearranged as

$$
\begin{gathered}
x(t)=\sum_{i=1}^{c} \mu_{i}(t)\left(\boldsymbol{\varphi}_{s}^{i}(t)\right)^{\mathrm{T}} \boldsymbol{\theta}_{i s} \\
y(t)=\sum_{i=1}^{c} \mu_{i}(t)\left[\left(\boldsymbol{\varphi}^{i}(t)\right)^{\mathrm{T}} \boldsymbol{\theta}_{i}+v(t)\right] .
\end{gathered}
$$

Since $\sum_{i=1}^{c} \mu_{i}(t)=1$, (9) is also rewritten as

$$
\begin{gathered}
y(t)=\sum_{i=1}^{c} \mu_{i}(t)\left(\boldsymbol{\varphi}_{s}^{i}(t)\right)^{\mathrm{T}} \boldsymbol{\theta}_{i s}+ \\
\sum_{i=1}^{c} \mu_{i}(t)\left(\boldsymbol{\varphi}_{n}(t)\right)^{\mathrm{T}} \boldsymbol{\theta}_{n}+v(t)= \\
\sum_{i=1}^{c} \mu_{i}(t)\left(\boldsymbol{\varphi}_{s}^{i}(t)\right)^{\mathrm{T}} \boldsymbol{\theta}_{i s}+\left(\boldsymbol{\varphi}_{n}(t)\right)^{\mathrm{T}} \boldsymbol{\theta}_{n}+v(t) .
\end{gathered}
$$

The whole parameter vector $\boldsymbol{\theta}$ and the system parameter vector $\boldsymbol{\theta}_{s}$ are defined as follows:

$$
\boldsymbol{\theta}:=\left[\begin{array}{c}
\boldsymbol{\theta}_{s} \\
\boldsymbol{\theta}_{n}
\end{array}\right] \in \mathbf{R}^{n+n_{d}}, \quad \boldsymbol{\theta}_{s}:=\left[\begin{array}{c}
\boldsymbol{\theta}_{1 s} \\
\boldsymbol{\theta}_{2 s} \\
\vdots \\
\boldsymbol{\theta}_{c s}
\end{array}\right] \in \mathbf{R}^{n} .
$$

In addition, the whole information vector $\boldsymbol{\Phi}(t)$ and the system information vector $\boldsymbol{\Phi}_{s}(t)$ are defined as follows:

$$
\begin{gathered}
\boldsymbol{\Phi}(t):=\left[\left(\boldsymbol{\psi}^{1}(t)\right)^{\mathrm{T}},\left(\boldsymbol{\psi}^{2}(t)\right)^{\mathrm{T}}, \ldots,\right. \\
\left.\left(\boldsymbol{\psi}^{c}(t)\right)^{\mathrm{T}},\left(\boldsymbol{\varphi}_{n}(t)\right)^{\mathrm{T}}\right]^{\mathrm{T}} \in \mathbf{R}^{n+n_{d}}, \\
n=n_{1}+n_{2}+\cdots+n_{c}, \quad n_{i}=n_{a}^{i}+n_{b}^{i}
\end{gathered}
$$

$$
\begin{aligned}
& \boldsymbol{\Phi}_{s}(t):=\left[\left(\boldsymbol{\psi}^{1}(t)\right)^{\mathrm{T}},\left(\boldsymbol{\psi}^{2}(t)\right)^{\mathrm{T}}, \ldots,\left(\boldsymbol{\psi}^{c}(t)\right)^{\mathrm{T}}\right]^{\mathrm{T}} \in \mathbf{R}^{n}, \\
& i=1,2, \ldots, c \\
& x=\left[\left(\boldsymbol{\psi}^{1}(t)\right)^{\mathrm{T}},\left(\boldsymbol{\psi}^{2}(t)\right)^{\mathrm{T}}, \ldots,\left(\boldsymbol{\psi}^{c}(t)\right)^{\mathrm{T}}\right]\left[\begin{array}{c}
\boldsymbol{\theta}_{1 s} \\
\boldsymbol{\theta}_{2 s} \\
\vdots \\
\boldsymbol{\theta}_{c s}
\end{array}\right]= \\
& \left(\boldsymbol{\Phi}_{s}(t)\right)^{\mathrm{T}} \boldsymbol{\theta}_{s} \\
& y(t)=\left[\left(\boldsymbol{\psi}^{1}(t)\right)^{\mathrm{T}}, \ldots,\left(\boldsymbol{\varphi}_{n}(t)\right)^{\mathrm{T}}\right]\left[\begin{array}{c}
\boldsymbol{\theta}_{1 s} \\
\boldsymbol{\theta}_{2 s} \\
\vdots \\
\boldsymbol{\theta}_{c s} \\
\boldsymbol{\theta}_{n}
\end{array}\right]+v(t)=
\end{aligned}
$$

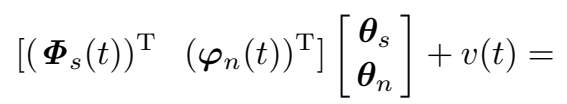

$$
\begin{aligned}
& (\boldsymbol{\Phi}(t))^{\mathrm{T}} \boldsymbol{\theta}+v(t) .
\end{aligned}
$$

For the system in (13), we use the minimum of the following objective function to confirm the parameters in (14) [20-22],

$$
J(\boldsymbol{\theta}):=\mathrm{E}\left\{\left\|y(t)-(\boldsymbol{\Phi}(t))^{\mathrm{T}} \boldsymbol{\theta}\right\|^{2}\right\} .
$$

Thus, the stochastic gradient (SG) identification algorithm can be used to estimate the unknown parameters of $(13)[23,24]$. However, because the whole information vector $\boldsymbol{\Phi}(t)$ of (13) contains unknown middle variables $x(t-j)$, unmeasurable noise terms $v(t-j)$ and uncertain label function $\mu_{i}(t)$, we cannot directly use the common $\mathrm{SG}$ algorithm to confirm the parameters.

For the label function values, an online fuzzy clustering method is proposed to estimate the label function values. First of all, the criterion function is built to judge which subsystem is activated. The unknown values $\mu_{i}(t)$ are replaced with their estimates $\widehat{\mu}_{i}(t)$ confirmed by the fuzzy clustering method, the unknown $x(t-j)$ is replaced with the outputs $x_{a}(t-j)$ of the generalized auxiliary model, and the unmeasurable noise terms $v(t-j)$ are replaced with their estimated residuals $\widehat{v}(t-i)$, respectively.

Define

$$
\begin{gathered}
\widehat{\boldsymbol{\Phi}}(t):=\left[\left(\widehat{\boldsymbol{\psi}}^{1}(t)\right)^{\mathrm{T}}, \ldots,\left(\widehat{\boldsymbol{\psi}}^{c}(t)\right)^{\mathrm{T}},\left(\widehat{\boldsymbol{\varphi}}_{n}(t)\right)^{\mathrm{T}}\right]^{\mathrm{T}}, \\
n=n_{1}+n_{2}+\cdots+n_{c}, \\
n_{i}=n_{a}^{i}+n_{b}^{i}, \quad\left\{\begin{array}{l}
\widehat{\mu}_{i}(t)=1, \widehat{\delta}_{t}=i \\
\widehat{\mu}_{i}(t)=0, \widehat{\delta}_{t} \neq i
\end{array}, \quad i=1,2, \ldots, c\right. \\
\widehat{\boldsymbol{\psi}}^{i}(t):=\widehat{\mu}_{i}(t)\left(\widehat{\boldsymbol{\varphi}}_{s}^{i}(t)\right)^{\mathrm{T}}=
\end{gathered}
$$




$$
\begin{gathered}
{\left[-\widehat{\mu}_{i}(t) x_{a}(t-1), \ldots, \widehat{\mu}_{i}(t) u\left(t-n_{b}^{i}\right)\right]^{\mathrm{T}}} \\
\widehat{\varphi}_{n}(t):=\left[\widehat{v}(t-1), \widehat{v}(t-2), \ldots, \widehat{v}\left(t-n_{d}\right)\right]^{\mathrm{T}} .
\end{gathered}
$$

Let $\widehat{\boldsymbol{\theta}}(t)=\left[\begin{array}{c}\widehat{\boldsymbol{\theta}}_{s}(t) \\ \widehat{\boldsymbol{\theta}}_{n}(t)\end{array}\right]$ be the estimate of $\boldsymbol{\theta}(t)=$ $\left[\begin{array}{l}\boldsymbol{\theta}_{s}(t) \\ \boldsymbol{\theta}_{n}(t)\end{array}\right]$ at time $t$. Here, we use the following generalized auxiliary model as the estimated model:

$$
x_{a}(t)=\left(\widehat{\boldsymbol{\Phi}}_{s}(t)\right)^{\mathrm{T}} \widehat{\boldsymbol{\theta}}_{s}(t) .
$$

The action of the generalized auxiliary model in (15) is like a real-time state observer or state estimator. By the aid of the generalized auxiliary model, we can estimate or observe the dynamic process of the unknown middle variables.

\section{MD based on fuzzy clustering}

For the MD, it can be usually considered as a clustering process. Therefore, we can use the fuzzy clustering method to establish the decision function. The following clustering objective function is given:

$$
J\left(\rho_{i}, \boldsymbol{\theta}_{i}\right)=\sum_{t=1}^{N} \sum_{i=1}^{c}\left(\rho_{i}(t)\right)^{q}\left[\frac{y(t)-\left(\widehat{\boldsymbol{\varphi}}_{s}^{i}(t)\right)^{\mathrm{T}} \boldsymbol{\theta}_{i s}}{\left\|\left[1,-\left(\boldsymbol{\theta}_{i s}\right)^{\mathrm{T}}\right]^{\mathrm{T}}\right\|}\right]^{2}
$$

where $\rho_{i}(t)$ represents the membership degree value of the actual output value $y(t)$ in the $i$ th mode, satisfying $\sum_{i=1}^{c} \rho_{i}(t)=1,1 \geqslant \rho_{i}(t) \geqslant 0 . \boldsymbol{\theta}_{i s}$ is the real parameter vector of the $i$ th subsystem. $q$ is the exponential weighting factor, and $N$ is the total number of samples.

Theorem 1 Given $\boldsymbol{\theta}_{i s}(i=1,2, \ldots, c)$ and a weighted factor $q$, then the minimum of the objective function in (16) can be obtained by satisfying the following condition.

$$
\rho_{i}(t)=\frac{1}{\sum_{j=1}^{c}\left[\frac{e_{i}(t)}{e_{j}(t)}\right]^{\frac{2}{(q-1)}}}, \quad i=1,2, \ldots, c
$$

where $e_{i}(t)=\frac{y(t)-\left(\widehat{\boldsymbol{\varphi}}_{s}^{i}(t)\right)^{\mathrm{T}} \boldsymbol{\theta}_{i s}}{\left\|\left[1,-\left(\boldsymbol{\theta}_{i s}\right)^{\mathrm{T}}\right]^{\mathrm{T}}\right\|}$.

Proof The objective function in (16) can be written as the following form:

$$
J\left(\rho_{i}\right)=\sum_{t=1}^{N} \sum_{i=1}^{c}\left(\rho_{i}(t)\right)^{q} e_{i}(t)^{2} .
$$

Due to $\sum_{i=1}^{c} \rho_{i}(t)=1$, we consider Lagrange multipliers for the objective function in (18), and have

$\bar{J}\left(\rho_{i}, \lambda\right)=\left[\sum_{t=1}^{N} \sum_{i=1}^{c}\left(\rho_{i}(t)\right)^{q} e_{i}(t)^{2}\right]+\sum_{t=1}^{N} \lambda\left[1-\sum_{i=1}^{c} \rho_{i}(t)\right]$.
Since $\rho_{i}(t)$ is independent of each other, the minimization of $\bar{J}\left(\rho_{i}, \lambda\right)$ on $\rho_{i}(t)$ is equivalent to the minimization of the following objective function on $\rho_{i}(t)$ :

$$
\widehat{J}\left(\rho_{i}\right)=\left(\rho_{i}(t)\right)^{q} e_{i}(t)^{2}+\lambda\left[1-\sum_{i=1}^{c} \rho_{i}(t)\right] .
$$

By using the derivative of the objective function in (20) on $\rho_{i}(t)$ and making it equal to zero, we have

$$
q\left(\rho_{i}^{*}(t)\right)^{q-1} e_{i}(t)^{2}-\lambda^{*}=0
$$

where $\rho_{i}^{*}(t)$ is the corresponding limit value. We can obtain

$$
\rho_{i}^{*}(t)=\left[\frac{\lambda^{*}}{q e_{i}(t)^{2}}\right]^{\frac{1}{q-1}} .
$$

Substituting (22) into $\sum_{i=1}^{c} \rho_{i}(t)=1$, there is the following result:

$$
\lambda^{*}(t)=\frac{1}{\sum_{j=1}^{c}\left(\frac{1}{q e_{j}(t)^{2}}\right)^{\frac{1}{q-1}} .}
$$

Substituting (23) into (22), (17) is achieved.

If each mode is considered as a cluster, Theorem 1 gives the distribution of the actual output in each cluster. By observing the clustering algorithm, when the estimated error $e_{i}(t)$ of the $i$ th mode is smaller, the membership degree value $\rho_{i}(t)$ of the $i$ th mode is bigger. This indicates that the current sampled output is more likely to belong to the $i$ th mode. Therefore, the membership degree functions of various modes can be used as the MD functions.

Based on Theorem 1, we use (17) as the decision function. The following criterion is employed to detect the mode value:

$$
\widehat{\delta}_{t}:=\underset{i=1,2, \ldots, c}{\arg } \max \rho_{i}(t)
$$

where

$$
\begin{gathered}
\rho_{i}(t)=\frac{1}{\sum_{j=1}^{c}\left[\frac{e_{i}(t)}{e_{j}(t)}\right]^{\frac{2}{(q-1)}}} \\
e_{i}(t)=\frac{y(t)-\left(\widehat{\boldsymbol{\varphi}}_{s}^{i}(t)\right)^{\mathrm{T}} \widehat{\boldsymbol{\theta}}_{i s}(t-1)}{\left\|\left[1,-\left(\widehat{\boldsymbol{\theta}}_{i s}(t-1)\right)^{\mathrm{T}}\right]^{\mathrm{T}}\right\|}, \quad i=1,2, \ldots, c
\end{gathered}
$$

where $\widehat{\boldsymbol{\theta}}_{i s}(t-1)$ and $\widehat{\boldsymbol{\varphi}}_{s}^{i}(t)$ are the estimates of $\boldsymbol{\theta}_{i s}(t-1)$ and $\varphi_{s}^{i}(t)$, respectively.

\section{Parameters estimation}

From (13), we get

$$
v(t)=y(t)-(\boldsymbol{\Phi}(t))^{\mathrm{T}} \boldsymbol{\theta} .
$$


Using the estimates $\widehat{\boldsymbol{\Phi}}(t)$ and $\widehat{\boldsymbol{\theta}}$ in place of $\boldsymbol{\Phi}(t)$ and $\boldsymbol{\theta}$ in the above equation, $\widehat{v}(t)$ can be calculated by

$$
\widehat{v}(t)=y(t)-(\widehat{\boldsymbol{\Phi}}(t))^{\mathrm{T}} \widehat{\boldsymbol{\theta}}(t) .
$$

Thus, the recursive gradient identification algorithm based on auxiliary model (RGA-AM) can be employed to confirm the parameters of (13).

$$
\begin{gathered}
\widehat{\boldsymbol{\theta}}(t)=\widehat{\boldsymbol{\theta}}(t-1)+\frac{\widehat{\boldsymbol{\Phi}}(t)}{r(t)} e(t) \\
e(t)=y(t)-\widehat{\boldsymbol{\Phi}}(t) \widehat{\boldsymbol{\theta}}(t-1) \\
r(t)=r(t-1)+\|\widehat{\boldsymbol{\Phi}}(t)\|^{2}, \quad r(0)=1 \\
x_{a}(t)=\left(\widehat{\boldsymbol{\Phi}}_{s}(t)\right)^{\mathrm{T}} \widehat{\boldsymbol{\theta}}_{s}(t) \\
\widehat{v}(t)=y(t)-(\widehat{\boldsymbol{\Phi}}(t))^{\mathrm{T}} \widehat{\boldsymbol{\theta}}(t)
\end{gathered}
$$

where

$$
\begin{aligned}
& \widehat{\boldsymbol{\Phi}}(t)=\left[\left(\widehat{\boldsymbol{\psi}}^{1}(t)\right)^{\mathrm{T}}, \ldots,\left(\widehat{\boldsymbol{\psi}}^{c}(t)\right)^{\mathrm{T}},\left(\widehat{\boldsymbol{\varphi}}_{n}(t)\right)^{\mathrm{T}}\right]^{\mathrm{T}} \\
& \widehat{\boldsymbol{\Phi}}_{s}(t)=\left[\left(\widehat{\boldsymbol{\psi}}^{1}(t)\right)^{\mathrm{T}},\left(\widehat{\boldsymbol{\psi}}^{2}(t)\right)^{\mathrm{T}}, \ldots,\left(\widehat{\boldsymbol{\psi}}^{c}(t)\right)^{\mathrm{T}}\right]^{\mathrm{T}} \\
& \left\{\begin{array}{l}
\widehat{\mu}_{i}(t)=1, \widehat{\delta}_{t}=i \\
\widehat{\mu}_{i}(t)=0, \widehat{\delta}_{t} \neq i
\end{array}, \quad i=1,2, \ldots c\right. \\
& \widehat{\boldsymbol{\psi}}^{i}(t)=\left[-\widehat{\mu}_{i}(t) x_{a}(t-1),, \ldots, \widehat{\mu}_{i}(t) u\left(t-n_{b}^{i}\right)\right]^{\mathrm{T}} \\
& \widehat{\varphi}_{s}^{i}(t)=\left[-x_{a}(t-1), \ldots,-x_{a}\left(t-n_{a}^{i}\right), u(t-1), \ldots,\right. \\
& \left.-u\left(t-n_{b}^{i}\right)\right]^{\mathrm{T}}, \\
& \widehat{\varphi}_{n}(t)=\left[\widehat{v}(t-1), \widehat{v}(t-2), \ldots, \widehat{v}\left(t-n_{d}\right)\right]^{\mathrm{T}} \\
& \widehat{\boldsymbol{\theta}}_{s}(t)=\left[\begin{array}{c}
\widehat{\boldsymbol{\theta}}_{1 s}(t) \\
\widehat{\boldsymbol{\theta}}_{2 s}(t) \\
\vdots \\
\widehat{\boldsymbol{\theta}}_{c s}(t)
\end{array}\right], \quad \widehat{\boldsymbol{\theta}}(t)=\left[\begin{array}{c}
\widehat{\boldsymbol{\theta}}_{s}(t) \\
\widehat{\boldsymbol{\theta}}_{n}(t)
\end{array}\right] \\
& \widehat{\boldsymbol{\theta}}_{i s}(t)=\left[\widehat{a}_{1}^{i}(t), \ldots, \widehat{a}_{n_{a}^{i}}^{i}(t), \widehat{b}_{1}^{i}(t), \ldots, \widehat{b}_{n_{b}^{i}}^{i}(t)\right]^{\mathrm{T}}, \\
& \widehat{\boldsymbol{\theta}}_{n}(t)=\left[\widehat{d}_{1}(t), \widehat{d}_{2}(t), \ldots, \widehat{d}_{n_{d}}(t)\right]^{\mathrm{T}} .
\end{aligned}
$$

The above-mentioned algorithm is known as the identification algorithm based on single innovation. According to the multi-innovation estimation principle [19-23], we construct a multi-innovation identification method based on the generalized auxiliary model (MI-GAM) to estimate the parameters.

Hence, we define an innovation vector

$$
\boldsymbol{E}(p, t)=\left[\begin{array}{c}
e(t) \\
e(t-1) \\
\vdots \\
e(t-p+1)
\end{array}\right] \in \mathbf{R}^{p}
$$

where the positive integer $p$ is the innovation length. From (27), we get

$$
e(t-j)=y(t-j)-(\widehat{\boldsymbol{\Phi}}(t-j))^{\mathrm{T}} \widehat{\boldsymbol{\theta}}(t-j-1) \in \mathbf{R} .
$$

Thus, the multi-innovation vector can be constructed as

$$
\boldsymbol{E}(p, t):=\left[\begin{array}{c}
y(t)-(\widehat{\boldsymbol{\Phi}}(t))^{\mathrm{T}} \widehat{\boldsymbol{\theta}}(t-1) \\
y(t)-(\widehat{\boldsymbol{\Phi}}(t-1))^{\mathrm{T}} \widehat{\boldsymbol{\theta}}(t-1) \\
\vdots \\
y(t)-(\widehat{\boldsymbol{\Phi}}(t-p+1))^{\mathrm{T}} \widehat{\boldsymbol{\theta}}(t-1)
\end{array}\right] \in \mathbf{R}^{p} .
$$

Furthermore, we define the following multi-innovation matrix $\widehat{\boldsymbol{\Xi}}(p, t)$ and stack output vector $\boldsymbol{Y}(p, t)$ as

$$
\begin{aligned}
\widehat{\boldsymbol{\Xi}}(p, t) & :=[\widehat{\boldsymbol{\Phi}}(t), \widehat{\boldsymbol{\Phi}}(t-1), \ldots, \widehat{\boldsymbol{\Phi}}(t-p+1)] \\
\boldsymbol{Y}(p, t) & :=[y(t), y(t-1), \ldots, y(t-p+1)]^{\mathrm{T}} .
\end{aligned}
$$

According to the multi-innovation estimation principle in [25-31], we achieve the MI-GAM algorithm with the innovation length $p$ as follows:

$$
\begin{gathered}
\widehat{\boldsymbol{\theta}}(t)=\widehat{\boldsymbol{\theta}}(t-1)+\frac{\widehat{\boldsymbol{\Xi}}(p, t)}{r(t)} \boldsymbol{E}(p, t) \\
\boldsymbol{E}(p, t)=\boldsymbol{Y}(p, t)-\widehat{\boldsymbol{\Xi}}(p, t) \widehat{\boldsymbol{\theta}}(t-1) \\
r(t)=r(t-1)+\|\widehat{\boldsymbol{\Phi}}(t)\|^{2}, \quad r(0)=1 \\
x_{a}(t)=\left(\widehat{\boldsymbol{\Phi}}_{s}(t)\right)^{\mathrm{T}} \widehat{\boldsymbol{\theta}}_{s}(t) \\
\widehat{v}(t)=y(t)-(\widehat{\boldsymbol{\Phi}}(t))^{\mathrm{T}} \widehat{\boldsymbol{\theta}}(t) .
\end{gathered}
$$

In order to understand the whole identification algorithm clearly, we summarize the whole algorithm as follows:

Step 1 Initialize all parameters, including the orders $n_{a}^{i}, n_{b}^{i}$ and $n_{d}, \widehat{\theta}(0)=1_{n+n_{d}} / p_{0}, p, q$ and $c$.

Step 2 The input vectors $\hat{\varphi}_{s}^{i}(t)(i=1,2, \ldots, c)$ are constructed by using (29) and the input signal. The estimated errors $e_{i}(t)(i=1,2, \ldots, c)$ are calculated by (24c). The fuzzy membership degree values $\rho_{i}(t)(i=$ $1,2, \ldots, c)$ of all modes are calculated by $(24 \mathrm{~b})$.

Step 3 According to (24a), the estimate of the label function of the selected mode where the membership degree value is the maximum is set to 1 , that is $\widehat{\mu}_{i}(t)=1$ (if $\widehat{\delta}_{t}=i$ ), and the estimates of other label functions are set to $0, \widehat{\mu}_{j}(t)=0(j \neq i, j=1,2, \ldots, s)$.

Step 4 The estimates $\widehat{\mu}_{i}(t), \ldots, \widehat{\mu}_{i}(t-p+1)$, the input vectors $\widehat{\varphi}_{s}^{i}(t), \ldots, \widehat{\boldsymbol{\varphi}}_{s}^{i}(t-p+1)(i=1,2, \ldots, c)$, and the residual vectors $\boldsymbol{\varphi}_{n}(t), \ldots, \boldsymbol{\varphi}_{n}(t-p+1)$ are collected to construct the whole information vectors $\widehat{\boldsymbol{\Phi}}(t), \ldots, \widehat{\boldsymbol{\Phi}}(t-$ $p+1)$.

Step 5 The algorithm in (31) - (35) is employed to estimate the parameters. 
Step 6 If $t=N, N$ is the total number of samples, then go to Step 7; otherwise, increment $t$ by 1 and go to Step 2.

Step 7 If $\left|\frac{J(k)-J(k-1)}{J(k)}\right| \leqslant \xi$, the algorithm is over; otherwise, $k=k+1$, go to Step 2 .

\section{Simulation}

In order to verify the effectiveness of the proposed method, the following switched system constructed from three linear subsystems and colored noises is studied herein,

$y(t)=\frac{B^{\delta_{t}}(z)}{A^{\delta_{t}}(z)} u(t)+D(z) v(t), \quad \delta_{t} \in i=\{1,2, \ldots, 3\}$

where

$$
\begin{gathered}
\text { Mode 1 }:\left\{\begin{array}{l}
A^{1}(z)=1-0.4 z^{-1}+0.25 z^{-2} \\
B^{1}(z)=-0.15 z^{-1}+0.08 z^{-2}
\end{array}\right. \\
\text { Mode 2 }:\left\{\begin{array}{l}
A^{2}(z)=1+z^{-1}+0.24 z^{-2} \\
B^{2}(z)=-0.65 z^{-1}+0.3 z^{-2}
\end{array}\right. \\
\text { Mode } 3:\left\{\begin{array}{l}
A^{3}(z)=1+1.55 z^{-1}-0.58 z^{-2} \\
B^{3}(z)=-2.1 z^{-1}+0.96 z^{-2}
\end{array}\right. \\
D(z)=1-z^{-1}+0.2 z^{-2} .
\end{gathered}
$$

Here, $\{u(t)\}$ is taken as a white Gaussian noise sequence with zero mean and unit variance. $\{v(t)\}$ is a white Gaussian noise sequence with zero mean and variance $\sigma^{2}$. The signal-to-noise ratio (SNR) is $25 \mathrm{~dB}$. For the design of the switched rule $\delta_{t}$, it is regarded as a piecewise constant function which is periodically switched from its current value to other values chosen from modes $\{1,2,3\}$. To test the effectiveness of the proposed algorithm, we adapt the different innovation lengths to identify the switched system, including $p=1,3,5$. For 1000 independent experiments for each innovation length, 1000 data sequences are generated by using the simulation example. In each experiment, the following fitness function is used to measure the model fitting situation.

$$
\mathrm{FIT}=\left(1-\frac{\|\widehat{\boldsymbol{y}}-\boldsymbol{y}\|_{2}}{\left\|\boldsymbol{y}-\overline{\boldsymbol{y}} \mathbf{1}_{N}\right\|_{2}}\right) \times 100 \%
$$

where $y$ and $\widehat{y}$ are the whole real outputs and the whole model outputs, respectively. $\bar{y}$ is the mean of the real outputs.

By using the proposed method, 1000 independent experiments with the different innovation lengths have been finished. The statistical results of independent experiments with $p=1,3,5$ are presented in Fig. 2, Fig. 3 and Fig. 4, respectively. The goodness range of fitness degree (GRFD) and average values (AV) are given in Table 1. From observing Figs. 2-4 and Table 1, the performance of the multiinnovation identification algorithm with $p \geqslant 2$ has higher accuracy than that of the single innovation identification algorithm with $p=1$, and the fitness average values of the parameter estimation become bigger and bigger as the innovation length $p$ increases. Simultaneously, with the increase of the innovation length $p$, the parameter estimation fitness average value is closer to one hundred percent.

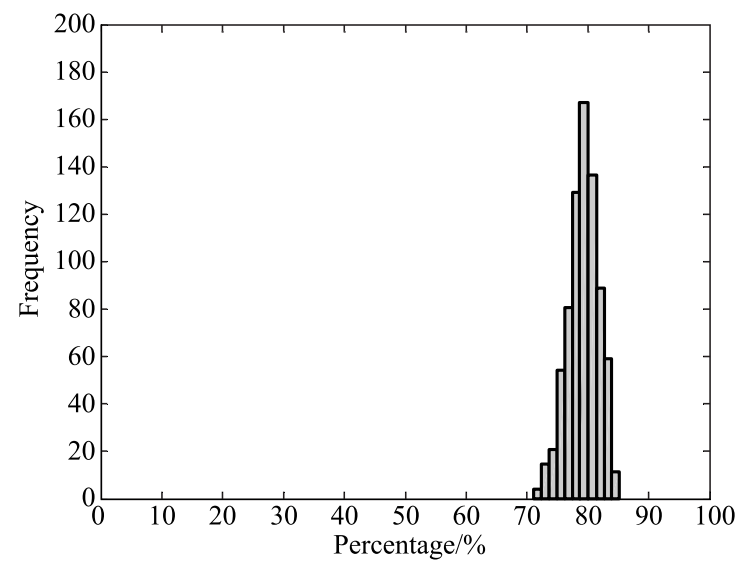

Fig. 2 Statistical result of independent experiments with $p=1$

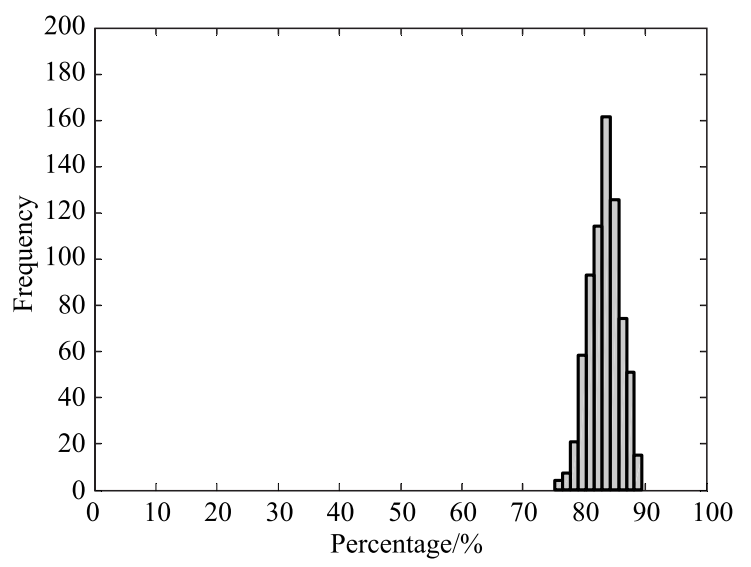

Fig. 3 Statistical result of independent experiments with $p=3$

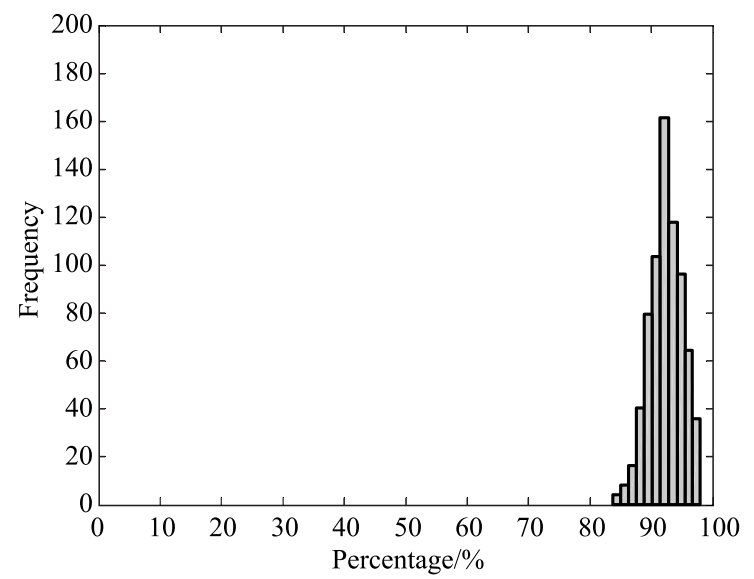

Fig. 4 Statistical result of independent experiments with $p=5$ 
In one independent experiment with the information length $p=5$, the identification results of three subsystems are shown in Figs. 5 - 7.

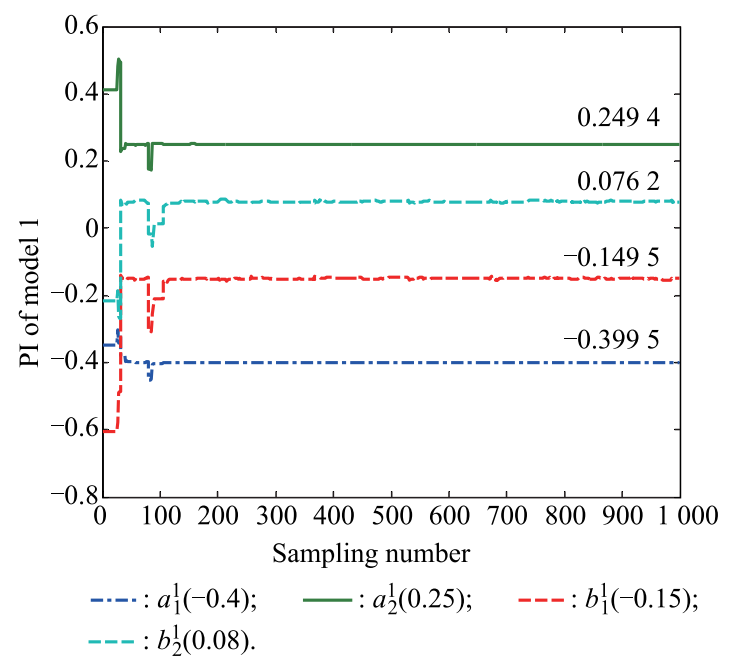

Fig. 5 Identification result of subsystem 1

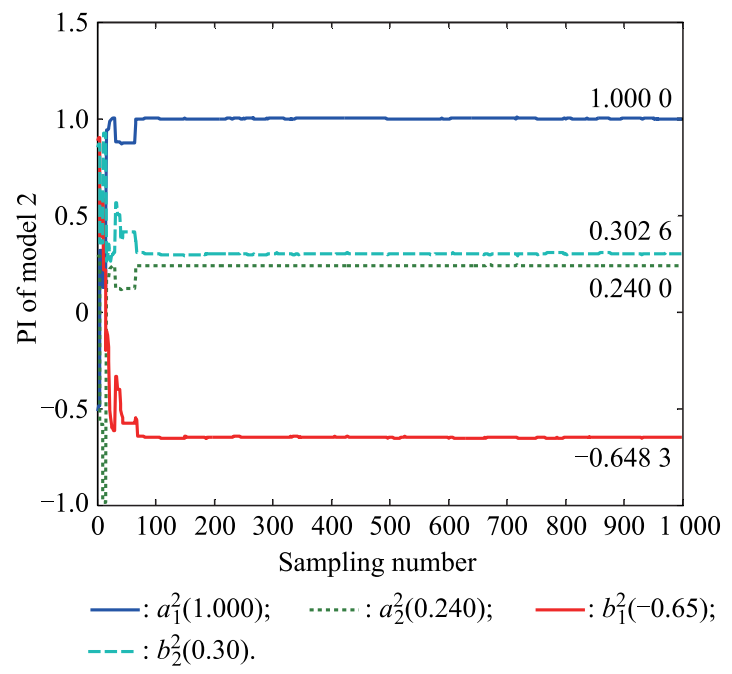

Fig. 6 Identification result of subsystem 2

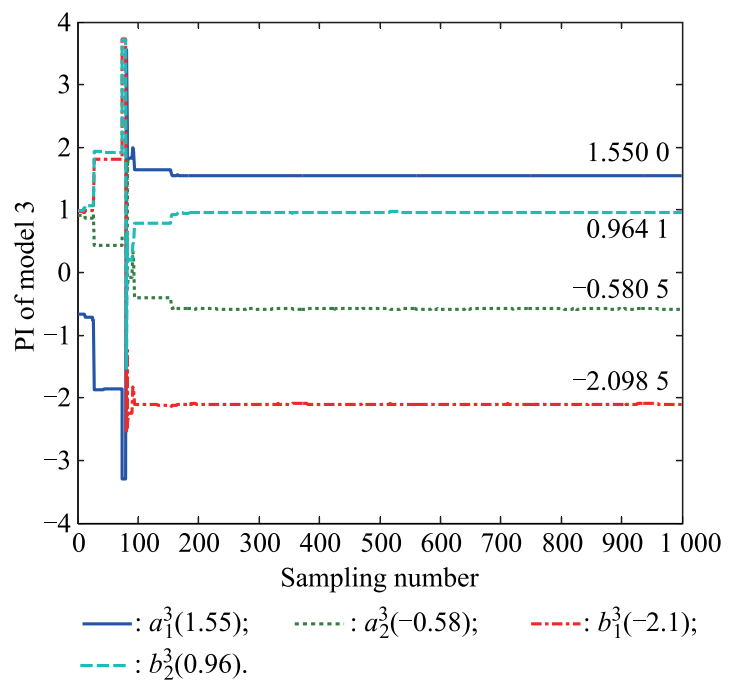

Fig. 7 Identification result of subsystem 3
Fig. 8 gives the parameters estimation process of the noise model. From Figs. 5-8, the estimated parameters are closer to the real parameters with the increase of the sampling steps.

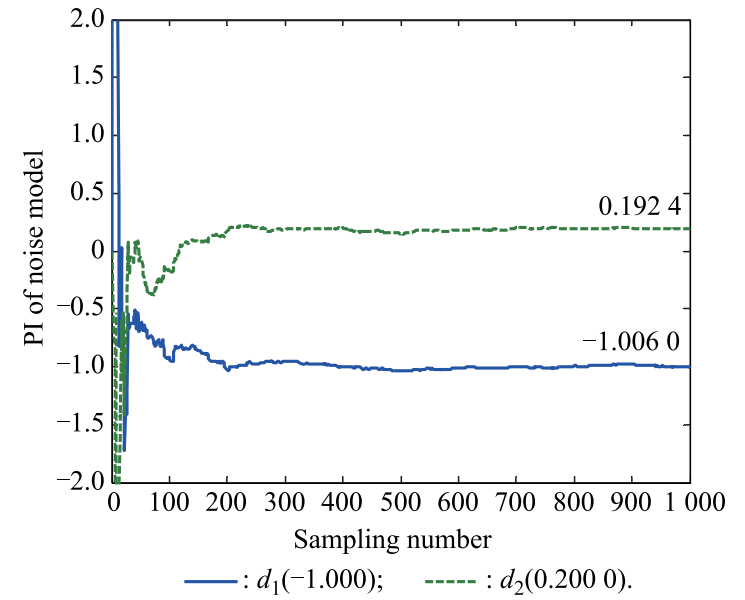

Fig. 8 Parameter estimation of noise model

Table 1 GRFD and AV

\begin{tabular}{cccc}
\hline $\begin{array}{c}\text { Information } \\
\text { length }\end{array}$ & $p=1$ & $p=3$ & $p=5$ \\
\hline GRFD & {$[71.5 \%, 86.6 \%]$} & {$[75.5 \%, 89.6 \%]$} & {$[84.5 \%, 98.6 \%]$} \\
AV & $80.8 \%$ & $83.8 \%$ & $94.7 \%$ \\
\hline
\end{tabular}

To illustrate the effectiveness of the proposed method, the performance of the proposed method is compared with that in [15]. The statistical results of estimated parameters with different identification methods are given in Table 2. In Table 2, the terms of the first column of each estimated parameter vector are $\mathrm{AV}$ and the terms of the second column of each estimated parameter vector are variances. From Table 2, the estimated AV with $p=5$ have higher accuracy than those in [15].

From Figs. 2-8, and Tables 1-2, we can get the following remarks:

(i) For the unknown variables in the switched linear system with colored noises, the auxiliary model method is used to estimate them. Thus, the parameters of the model are estimated by the multi-innovation gradient identification method. From the simulation results, the proposed identification algorithm becomes simple, concise and effective.

(ii) The fuzzy clustering method based on the input/output data captures the MD process very well. Especially, the proposed fuzzy clustering method is used to deal with the mode detection by an on-line way. 
Table 2 Statistical results based on different identification algorithms

\begin{tabular}{ccc}
\hline Model & The algorithm in $[15]$ & Our algorithm $(p=5)$ \\
\hline Model 1 & $\widehat{\boldsymbol{\theta}}_{1}=\left[\begin{array}{c}-0.398 \pm 0.019 \\
0.251 \pm 0.024 \\
-0.148 \pm 0.052 \\
0.081 \pm 0.015\end{array}\right]$ & $\widehat{\boldsymbol{\theta}}_{1}=\left[\begin{array}{c}-0.4001 \pm 0.003 \\
0.2498 \pm 0.002 \\
-0.1487 \pm 0.001 \\
0.0801 \pm 0.003\end{array}\right]$ \\
\hline Real parameter: $\boldsymbol{\theta}_{1}=[-0.4,0.25,-0.15,0.08]^{\mathrm{T}}$ & \\
\hline Model 2 & $\widehat{\boldsymbol{\theta}}_{2}=\left[\begin{array}{c}0.983 \pm 0.0378 \\
0.2355 \pm 0.0246 \\
-0.649 \pm 0.0371 \\
0.2987 \pm 0.0415\end{array}\right]$ & $\widehat{\boldsymbol{\theta}}_{2}=\left[\begin{array}{c}0.9996 \pm 0.002 \\
0.2394 \pm 0.003 \\
-0.6504 \pm 0.003 \\
0.2998 \pm 0.004\end{array}\right]$ \\
\hline Real parameter: $\boldsymbol{\theta}_{2}=[1,0.24,-0.65,0.3]^{\mathrm{T}}$ & {$\left[\begin{array}{c}1.5505 \pm 0.0012 \\
-0.579 \pm 0.0026 \\
-2.101 \pm 0.0042 \\
0.9596 \pm 0.0030\end{array}\right]$} \\
\hline Model 3 & $\widehat{\boldsymbol{\theta}}_{3}=\left[\begin{array}{c}1.5577 \pm 0.025 \\
-0.5716 \pm 0.038 \\
-2.0768 \pm 0.071 \\
0.9645 \pm 0.0449\end{array}\right]$ & $\widehat{\boldsymbol{\theta}}_{3}=\left[\begin{array}{c}-0.9855 \pm 0.015 \\
0.1926 \pm 0.012\end{array}\right]$ \\
\hline Real parameter: $\boldsymbol{\theta}_{3}=[1.55,-0.58,-2.1,0.96]^{\mathrm{T}}$ \\
\hline $\boldsymbol{D}=\left[\begin{array}{c}-0.949 \pm 0.09 \\
0.159 \pm 0.04\end{array}\right]$
\end{tabular}

\section{Conclusions}

In our paper, we propose a modeling algorithm to solve the identification issues of switched linear systems with colored noises. In practical switched systems, there exist a lot of nonlinear systems. The identification of switched nonlinear systems is still difficult. The difficult problems are mainly embodied as follows:

i) What models are chosen to describe switched nonlinear systems;

ii) MD of switched nonlinear systems is a very difficult problem;

iii) Parameter estimation and convergence performance analysis are very difficult problems.

The modeling issue of switched nonlinear systems is a future direction of our research.

\section{References}

[1] OSUKA A, MATSUOKA Y, YSUTSU T, et al. Development of pinion-assist type electric power steering system. KOYO Engineering Journal, 2002, 161(1): $567-578$.

[2] GOLLU A, VARAIYA P D. Hybdrid dynamic systems. Proc. of the 28th IEEE Conference on Decision and Control, 1989: $2708-2713$

[3] SEIDMAN T I. Switching systems. Baltimore, the United States: UMBC, 1999.

[4] FERRARI-TRECATE G, MUSELLI M, LIBERATI D, et al. A clustering technique for the identification of piece wise affine systems. Automatica, 2003, 39(2): 205-217.

[5] BEMPORAD A, GARULLI A, PAOLETTI S, et al. A bounded error approach to piecewise affine system identification. IEEE Trans. on Automatic Control, 2005, 50(10): 1567 1580 .
[6] MA Y, RENÉ V. Identification of deterministic switched ARX systems via identification of algebraic varieties. Proc. of the 8th International Workshop on Hybrid Systems: Computation and Control, 2005: 449-465.

[7] VIDAL R, SOATTO S, MA Y, et al. An algebraic geometric approach to the identification of a class of linear hybrid systems. Proc. of the 42nd IEEE Conference on Decision and Control, 2003: $167-172$.

[8] ROLL J, BEMPORAD A, LJUNG L. Identification of piece wise affine systems via mixed-integer programming. Automatica, 2004, 40(1): $37-50$.

[9] JULOSKI A, WEILAND S. A Bayesian approach to the identification of piecewise linear output error models. Proc. of the 14th IFAC Symposium on System Identification, 2006: $374-$ 379.

[10] JULOSKI A, WEILAND S, HEEMELS W. A Bayesian approach to identification of hybrid systems. IEEE Trans. on Automatic Control, 2005, 50(10): 1520-1533.

[11] VERDULT V, VERHAEGEN M. Subspace identification of piecewise linear systems. Proc. of the 43rd IEEE Conference on Decision and Control, 2004: 3838 - 3843.

[12] BORGES J, VERDULT V, VERHAEGEN M, et al. A switching detection method based on projected subspace classification. Proc. of the 44th IEEE Conference on Decision and Control, and the European Control Conference, 2005: 344-349.

[13] PEKPE K, MOEROT G, GASSO K, et al. Identification of switching systems using change detection technique in the subspace framework. Proc. of the 43rd IEEE Conference on Decision and Control, 2004: 3720-3725.

[14] VIDAL R. Recursive identification of switched ARX systems. Automatica, 2008, 44(9): 2274-2287.

[15] LAURENT B, KHALED B, ERIC D, et al. A recursive identification algorithm for switched linear/affine models. Nonlinear Analysis: Hybrid Systems, 2011, 5(1): 242-253.

[16] LAURENT B. Identification of switched linear systems via sparse optimization. Automatica, 2011, 47(2): 668-677.

[17] WANG J D, CHEN T W. Online identification of switched linear output error models. Proc. of the IEEE International Sym- 
posium on Computer-Aided Control System Design, 2011: $1379-1384$.

[18] DING F, WANG Y. Filtering-based iterative identification for multivariable systems. IET Control Theory \& Applications, 2016, 10(8): 894-902.

[19] WANG H W, XIE L R. Convergence analysis of a least squared algorithm of linear switched identification. Journal of Control and Decision, 2019, 6(3): 1-12.

[20] CHEN J, XIONG J. Switching time estimation for switching ARMAX systems. Telecommunication Engineering, 2017, 57(9): $1011-1016$.

[21] DING F. Systems identification-performance analysis of the identification methods. Beijing: Science Press, 2014.

[22] DING F, CHEN T W. Performance analysis of multiinnovation gradient type identification methods. Automatica, 2007, 43(1): 1- 14.

[23] DING F, CHEN T W. Multi-innovation stochastic gradient identification methods. Proc. of the 16th World Congress on Intelligent Control and Automation, 2016: $1501-1505$.

[24] MAO Y, DING F, YANG E. Adaptive filtering-based multiinnovation gradient algorithm for input nonlinear systems with autoregressive noise. International Journal of Adaptive Control and Signal Processing, 2017, 31(3): 1388-1400.

[25] LI X L, ZHOU L C, DING R F, et al. Recursive least squares estimation algorithm for Hammerstein nonlinear systems with non-uniform sampling. Mathematical Problems in Engineering, 2013, 67(9): $1811-1818$.

[26] ZHENG E X, LIU R R, JIANG Y F, et al. Stochastic gradient identification for Hammerstein systems with non-uniformly sampling. Computer Systems Science and Engineering, 2016, 31(6): 439-444.

[27] LIU R R, ZHENG E X, CHANG S, et al. Hierarchical stochastic gradient identification for non-uniformly sampling Hammerstein systems with colored noise. Computer Systems Science and Engineering, 2016, 31(6): 425-430.
[28] ZHOU L C, LI X L, PAN F. Gradient-based iterative identification for wiener nonlinear systems with non-uniform sampling. Nonlinear Dynamics, 2014, 76 (1): 627-634.

[29] LIU R R, LI H R, PAN T H, et al. Parameter estimation for non-uniformly sampled wiener systems with dead-zone nonlinearities. IFAC-Papers On-Line, 2015, 28(8): 789-794.

[30] ZHOU L C, LI X L, SHAN L J, et al. Hierarchical recursive least squares parameter estimation of non-uniformly sampled Hammerstein nonlinear systems based on Kalman filter. Journal of the Franklin Institute, 2017, 354(10): 4231 - 4246.

[31] LIU R R, PAN T H, LI Z M. Multi-model recursive identification for nonlinear systems with non-uniformly sampling. Cluster Computer, 2017, 20(11): 25-32.

\section{Biographies}

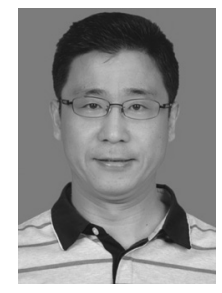

WANG Hongwei was born in 1969. He is a Ph.D. and a professor. He was sent to do discipline construction work in Xinjiang University by Dalian University of Technology. His research interests are switched system identification, nonlinear system identification, and fuzzy modeling.

E-mail: 1195201627@qq.com

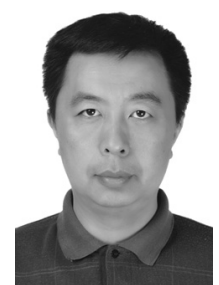

XIA Hao was born in 1971. He is a Ph.D. and a professor in Dalian University of Technology. His research interests are industrial modeling, logistics control and simulation.

E-mail: hao.x.xia@dlut.edu.cn 